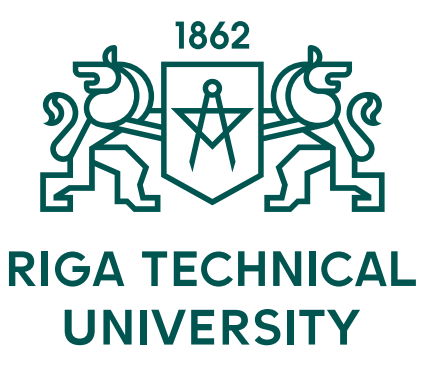

Sabīne Upnere

\title{
INVESTIGATION OF DYNAMICS AND RELIABILITY OF HIGH-POWERED WATER-COOLING SYSTEMS
}

Summary of the Doctoral Thesis

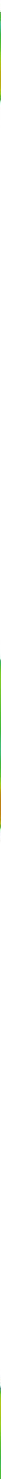

RTU Press

Riga 2019 
RIGA TECHNICAL UNIVERSITY

Faculty of Mechanical Engineering, Transport and Aeronautics

Institute of Mechanics and Mechanical Engineering

\section{Sabīne Upnere}

Doctoral Student of the Study Programme "Engineering, Mechanics and Mechanical

Engineering"

\section{INVESTIGATION OF DYNAMICS AND \\ RELIABILITY OF HIGH-POWERED WATER-COOLING SYSTEMS}

Summary of the Doctoral Thesis

Scientific supervisor

Professor Dr. sc. ing. JĀNIS AUZIN̦Š 
Upnere, S. Investigation of Dynamics and Reliability of High-Powered Water-Cooling Systems. Summary of the Doctoral Thesis. Riga: RTU Press, 2019. 32 p.

Published in accordance with the decision of the Promotion Council "P-04" of 7 November 2019, Minutes No. 10.

The Doctoral Thesis was partially developed with the financial support of the Latvian Council of Science project "Creation of design of experiments and metamodeling methods for optimization of dynamics of multibody 3D systems interacting with bulk solids and fluids" (lzp-2018/2-0281) and Doctoral Research Grant of the Faculty of Mechanical Engineering, Transport and Aeronautics of Riga Technical University. 


\section{DOCTORAL THESIS PROPOSED TO RIGA TECHNICAL UNIVERSITY FOR PROMOTION TO THE SCIENTIFIC DEGREE OF DOCTOR OF ENGINEERING SCIENCES}

To be granted the scientific degree of Doctor of Engineering Sciences, the present Doctoral Thesis has been submitted for the defence at the open meeting of RTU Promotion Council

on

December 20, 2019 at the Faculty of Mechanical Engineering, Transport and Aeronautics of Riga Technical University, Kipsalas street 6B, room .....

\section{OFFICIAL REVIEWERS}

Professor PhD Staffan Lundström

Luleå University of Technology, Sweden

Professor Dr. habil. sc. phys. Juris Roberts Kalniņš

Ventspils University of Applied Sciences, Latvia

Professor Dr. habil. sc. ing. Jānis Vība

Riga Technical University

\section{DECLARATION OF ACADEMIC INTEGRITY}

I hereby declare that the Doctoral Thesis submitted for review to Riga Technical University for the promotion to the scientific degree of Doctor of Engineering Sciences is my own. I confirm that this Doctoral Thesis had not been submitted to any other university for the promotion to a scientific degree.

Sabīne Upnere (signature)

Date:

The Doctoral Thesis has been written in Latvian. It consists of Introduction; 4 chapters; Conclusions; 65 figures; 9 tables; the total number of pages is 123. The Bibliography contains 149 titles. 


\section{TABLE OF CONTENTS}

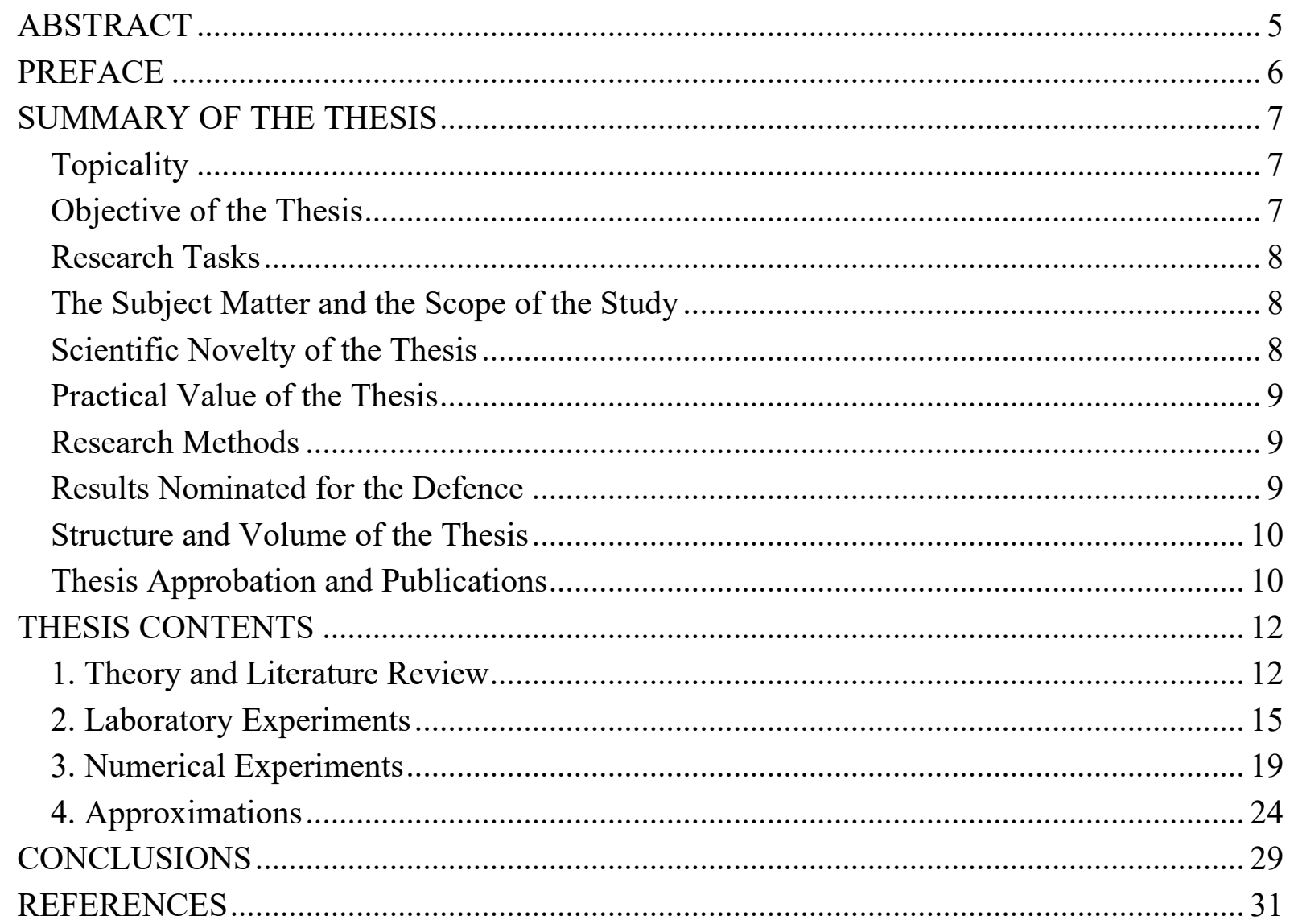




\begin{abstract}
The Thesis is dedicated to flow-induced vibration studies in high-powered cooling systems consisting of many rigid rods between which water or heavy water $\left(\mathrm{D}_{2} \mathrm{O}\right.$ - deuterium oxide) flows as a cooling agent. The goal is to determine the impact on dominant frequencies of the two system parameters - rod mass and its mounting stiffness. Due to simplicity, the investigated model system consists of a single, flexibly mounted rod inserted into an array of rigid rods with a triangular arrangement. The moving rod has two degrees of freedom. Both ends of it may move in the direction of flow and perpendicular to the flow. Several series of laboratory experiments have been performed to study in depth the impact of the single moving rod mass, the position in the array and the support stiffness on the amplitude and frequency of oscillations at different flow rates. The experiments were conducted in collaboration with the Paul Scherrer Institute in Switzerland. An analysis of the obtained data showed that the frequency ratio of vibrations caused by natural frequency and turbulence is a parameter that allows detecting changes in the above-mentioned input parameters in indirect way. Computational fluid dynamics (CFD) simulations were performed to study numerically the behaviour of a flexibly mounted rod in the cross-flow. To model the rod movement, it was described as a mass - spring system. In addition, it was analysed how to minimise computing time and costs. The optimal size of the computational domain was found for the given Reynolds numbers. Simulations at different inflow velocities allowed to analytically describe the distribution of the flow field in the gap between two adjacent cylinders introducing inflow boundary conditions for CFD simulations. The impact of the mass and the stiffness coefficient of the flexibly mounted rod on frequencies of the oscillation was assessed using the metamodeling approach. Using data from laboratory and numerical experiments, new secondorder and 7-term orthonormal Legendre polynomial approximation models were created. The inverse analysis, which was applied to experimental data, allowed to develop a model describing the relationship between the frequency ratio, the stiffness and the mass. The created approximation models are applicable to the alleviation of uncertainty, monitoring of cooling systems or early detection of damage related to the reduction of the mass of rods or to changes in the stiffness of the supports.
\end{abstract}




\section{PREFACE}

This work has been carried out in the Institute of Mechanics and Mechanical Engineering of Riga Technical University. Laboratory experiments were organised together with the Paul Scherrer Institute (PSI) in Switzerland.

Several people have been important in development of this work.

First, I would like to express my gratitude to my supervisor Professor Jānis Auziņ̌ for his guidance and support throughout the work on the Thesis.

Special thanks go to Dr Normunds Jēkabsons for his suggestions and cooperation during several years.

Particular gratitude goes to Dr Sergejs Dementjevs from PSI. His great work capability has always been inspiring. I would like to sincerely thank Phillipe Barbagallo (PSI) for the development of experimental installations. My gratitude to Professor Daniela Kiselev (PSI) and Dr Michael Wohlmuther (PSI).

Thanks to my colleagues in the Institute of Mechanics and Mechanical Engineering, especially Professor Jānis Vība and Professor Andrejs Krasnikovs.

Thanks to Professor Roberts Joffe of Luleå Technical University for the opportunity to always ask and get useful tips.

I would like to thank Paul Scherrer Institute for financing laboratory experiments. Thanks to Ventspils University of Applied Sciences for the permission to use the high-performance computational cluster.

Many thanks to my family and friends for their encouragement and belief that I will succeed. I would like to express my sincere thanks to my Friend for his patience and continuous support.

Riga October 2019

Sabīne Upnere 


\section{SUMMARY OF THE THESIS}

\section{Topicality}

As the technological capabilities for growing the productivity (capacity) of cooling equipment increase, the requirements for safety systems for this new equipment, which must be able to identify potential problems even before an emergency occurs, are also increasing. This is particularly important in new installations whose operational interruption or malfunction could have serious consequences for both the economy and the environment. Such modern cooling systems are used both in energy and in the cooling of various technological equipment in industry. This kind of equipment with heavy water is used in nuclear reactors and related devices.

Due to extensive use in various fields of engineering, the flow around tubes and rods is being studied intensively, but there are still a number of physical phenomena that are not sufficiently understood and investigated, such as 1) turbulence, 2) cavitation, 3) various aspects of the fluid-structure interaction problem, 4) finding global bifurcation of motion modes for vibrating systems, etc. Vibrations caused by turbulent flow in cooling systems, heat exchangers or nuclear power plants are considered to be one of the most devastating problems.

High-speed flow interaction with structural elements may result in vibrations of components, which, in turn, may lead to fatigue damage, rod collisions or connection damage, etc. For the safe operation of engineering equipment, it is necessary to continuously monitor whether the system does not exceed its operational limits and does not compromise its structural integrity. At the same time, it is not possible to measure changes in the internal elements (parameters) of the system directly during the operation of the equipment. For complex systems, its behaviour as a function of the input parameters is generally not known. In such situations, physical and numerical experiments can often be the only way to determine the relevant relationships. Adequate mathematical models can be used to detect as early as possible changes in the typical behaviour of the system that could indicate its malfunction and structural integrity problems. This Thesis examines the effects of changes in two parameters - the mass and the support stiffness of the rod.

\section{Objective of the Thesis}

The objective of the work is to explore the dynamics of the high-capacity water cooling system as a common system of fluid (water) and discrete rods, with a view to creating a new mathematical approximation models applicable for the design and monitoring of new systems with guaranteed functioning safety. 


\section{Research Tasks}

The main tasks of the present Thesis are as follows:

1) to carry out an overview the existing theories and practices for the identification of dynamic systems with a view to develop output data for laboratory experiments for rod vibrations in fluid environments;

2) to develop laboratory experimental procedures and design of experiments for rods in fluid environments;

3) to carry out laboratory experiments in collaboration with the Paul Scherrer Institute, PSI;

4) to carry out post-processing and analysis of experimental data with a view to implement further numerical experiments;

5) to plan numerical experiments, their implementation, approximation of results and to obtain an impact assessment of system parameters (criterion);

6) to develop water-cooling system approximation models that are applied for monitoring of systems where a reduction in the mass of the rods or a change in support stiffness during operation is expected;

7) to verify and validate the developed models;

8) to develop inverse approximation models for the safety and monitoring of the operation of cooling systems.

\section{The Subject Matter and the Scope of the Study}

The subject matter of the study is a system of triangular arrangements of closely located rods through which turbulent water flows. The scope of the study is system parameters: flow rate, vibrating rod mass, support stiffness coefficient, the position of the vibrating rod in the array and hydrodynamic force that acts on the rod.

\section{Scientific Novelty of the Thesis}

1. A large set of measurement data has been obtained for the amplitude and characteristic frequencies of the elastically-fixed rod at the various initial conditions of the system, using laboratory experiments for the closely-packed rod bundle.

2. Analysis of laboratory experiment data identified the parameter that allows the most effective detection of changes in the mass and support stiffness of a flexibly mounted rod.

3. A typical flow field distribution profile between two adjacent rods in an array with closely-packed rods has been found and mathematically approximated. The profile is applicable to Computational Fluid Dynamics simulations as the boundary condition, allowing a significant reduction in computing time and costs.

4. The inverse analysis results in mathematical approximations for the description of relationships between a) the rod support stiffness and the frequency ratio, and b) the 
rod mass and the frequency ratio. The inverse model allows assessing changes in the behaviour of the system over time, knowing its inherent frequencies.

\section{Practical Value of the Thesis}

The results of the study are applicable to the development and monitoring of widespectrum equipment, where fast flowing water is used to cool objects. In Latvia, the results of the study are applicable, for example, to industrial installations where fast cooling of the object is required. Another possible use is in cooling plants in thermal installations. In addition, the conclusions obtained are applicable in the monitoring system for the PSI proton beam target SINQ (Swiss Spallation Neutron Source).

The summarized results are useful in the development of analytical models that would describe the interaction of a flexibly mounted rod and flow in an array of closely placed rods. The resulting conclusions on the impact of the size of computational domain on the outcomes, and velocity profile can be used to create simplified numerical models without significantly losing accuracy while saving on computing resources.

The resulting approximation models allow to create monitoring systems and software for safe monitoring of parameters that cannot be measured directly.

\section{Research Methods}

The following methods were used to obtain comprehensive information on the parameters of the cooling plant consisting of an array of rods through which the water flows:

1) method of applying classical mechanical oscillation theory;

2) method of laboratory experiments of physical process in fluid (water) environments;

3) method of processing experimental data;

4) numerical modelling with computer methods;

5) metamodeling theory and methods.

\section{Results Nominated for the Defence}

1. Calculation methodology for efficient numerical modelling of the flow through a closely packed array of rods, including the selection of an optimal computing domain and an approximated flow velocity profile in a gap between two adjacent rods.

2. The inverse approximation model, which describes the dependency of the stiffness of the rod support from characteristic frequency ratio, which can be used in the safety monitoring of the system.

3. The inverse metamodel describing the mass of oscillating rod as a function of two parameters, the frequency ratio and the support stiffness, which can be applied for determining the rod damage associated with reducing the mass of the rod. 


\section{Structure and Volume of the Thesis}

The promotion work consists of an introduction, 4 chapters, conclusions and a list of references. The work comprises 123 pages, 65 images, 9 tables and a list of literature that contains 149 titles.

\section{Thesis Approbation and Publications}

The results of the Thesis have been reported at the following international conferences:

- 8th International Conference on Mathematical Modeling in Physical Sciences, Bratislava, Slovakia, August 26-29, 2019.

- 1 st International Conference on Numerical Modelling in Engineering, Gent, Belgium, August 28-29, 2018.

- 4th and 3rd International Conference on Optimization and Analysis of Structures, Tartu, Estonia, August 22-23, 2018, and August 23-25, 2015, respectively.

- 29th International Conference "Vibroengineering", Vilnius, Lithuania, December 1, 2017.

- 3rd International Conference "Innovative Materials, Structures and Technologies", Riga, Latvia, September 28-29, 2017.

- International Conference on Engineering Vibration, Sofia, Bulgaria, September 4-7, 2017.

- 16th and 15th International Scientific Conference on Engineering for Rural Development, Jelgava, Latvia, May 24-26, 2017, and May 25-27, 2016, respectively.

- 5th European Seminar on Computing, Plzen, the Czech Republic, Jun 5-10, 2016. Received the Award of the Best Poster.

- International Symposium and Winter-School on Modeling, Adaptive Discretizations and Solvers for Fluid-Structure Interaction, Linz, Austria, January 11-15, 2016.

The main results of the work are set out in 10 scientific publications in journals and reviewed conference articles. All articles are indexed in Scopus and/or Web of Sciences databases.

1. Upnere, S., Auzins, J., Jekabsons, N. Metamodel-Based Analysis of Cross-FlowInduced Vibrations. Lecture Notes in Mechanical Engineering, 2019, Springer. https://doi.org/10.1007/978-981-13-2273-014

2. Upnere, S., Jekabsons, N., Dementjevs, S., Wohlmuther, M. Experimental Studies of a Single Flexibly-Mounted Rod in a Triangular Rod Bundle in Cross-Flow. MATEC Web of Conferences, 2018, Vol. 148, 09002. https://doi.org/10.1051/matecconf/201814809002

3. Upnere, S. Numerical Study of Flow-Induced Vibrations of Multiple FlexiblyMounted Cylinders in Triangular Array. Latvian Journal of Physics and Technical Sciences, 2018, No. 5. https://doi.org/10.2478/lpts-2018-0035 
4. Upnere, S., Jekabsons, N. Stability Evaluation of the Rod in Triangular Array Predicted by CFD. IOP Conference Series: Materials Science and Engineering, 2018, ISSN 1757-8981. e-ISSN 1757-899X. https://doi.org/10.1088/1757$899 \mathrm{X} / 251 / 1 / 012062$

5. Upnere, S., Jekabsons, N. Dynamic Displacement Estimation Using Data Fusion. Vibroengineering PROCEDIA, 2017, Vol. 15, pp. 145-149. ISSN 2345-0533. https://doi.org/10.21595/vp.2017.19425

6. Upnere, S., Jekabsons, N. Estimation of Cross-Flow Influence on Spring-Mounted Cylinder in Triangular Cylinder Array. Proc. 16th International Scientific Conference “Engineering for Rural Development”, 2017, pp. 792-797. ISSN 1691-5976.

7. Upnere, S., Jekabsons, N. Flow-Induced Vibration of Cylindrical Structures. Proc. 15th International Scientific Conference "Engineering for Rural Development", 2016. pp. 01244-1249. ISSN 1691-5976.

8. Upnere, S. Modeling of the elastically-mounted cylinder behavior in the cross-flow. Accepted for publishing in Journal of Theoretical and Applied Mechanics, Vol. 49, pp. 327-342, 2019.

9. Upnere, S. Numerical Investigation of Flow-Induced Forces in the Rods Bundle. Accepted for publishing in IOP Journal of Physics: Conference Series, 2019.

10. Upnere, S., Jekabsons, N., Dementjevs, S., Wohlmuther M. Effects of variable parameters on the behaviour of the single flexibly-mounted rod in a closely-packed array. Accepted for publishing in Journal of Vibroengineering, 2019-2020. 


\section{THESIS CONTENTS}

\section{Theory and Literature Review}

Chapter 1 devoted to a brief overview of scientific literature and basic theory issues concerning: 1) flow-induced vibrations (FIV) in tube or rod systems; 2) Computational Fluid Dynamics (CFD) methods to predict aspects of vibration generation in arrays under different initial conditions; and 3) the possibilities of metamodelling in the planning of experiments, the exploration and approximation of mechanical systems.

The arrays of rods in commercial heat exchangers and water-cooling systems are made up of simple geometric patterns - squares or triangles [1]. Efficient cooling structures often require high flow speeds, as well as reduction of tube wall thickness, while taking into account vibration mechanisms that may result from flow in the array. The FIV problem thus becomes one of the most important dynamic challenges in the design process of cooling systems.

The excitation mechanisms, which create oscillations in the array of rods, are directly related to the flow rate. When the flow has reached the velocity required for the transition to turbulence, the nature of the flow causes a random dynamic pressure on the surface of the rod, including the random movement of the rod. As the flow rate continues to grow, it also causes the vortex to break from the surface as it flows through the array of rods, resulting in harmonious variations of pressure. At high flow velocities, there is an interaction between the vibrations of the flow field and the array of rods. Three basic mechanisms are defined classically, which cause a structural element to vibrate due to the cross-flow through the rod array. These are - vortex shedding, turbulent buffeting and fluidelastic instability [2]. The typical behaviour of the array of rods also depends on mechanical and geometric features, such as the method of fixing the rod and the configuration in the array, the pitch-to-diameter ratio $(P / d)$, etc.

The work of the present Thesis was focused on studies of the vibrations caused by turbulence in a rod bundle with closely packed cylindrical rods. An example of such system could be SINQ target. SINQ is a proton beam target located in Paul Scherrer Institute (Switzerland). The key element of the target is a bundle of rods (315 pieces in 37 rows) fabricated from $\mathrm{Zr}$ alloy tubes and filled with $\mathrm{Pb}$. Due to the way of mounting of the cylindric rods in the target, see Fig. $1.1 \mathrm{a}$, it is expected that the rod can move within a small tolerance range in the plane perpendicular to the axis of the cylinder. A proton beam initiates a spallation reaction, which is accompanied by a powerful heat release in the rods. The rods are cooled by turbulent heavy water cross flow that can induce the vibrations of the rod. Parameters of the vibrations depend on the rigidity of structure, geometry of the flow path, properties of heavy water, and flow rate. The SINQ target is well designed, but the total power has tremendously increased over the years. Therefore, in specific cases, it is possible that there occur damages in the rod because of water boiling, crack of the pipe and lead leakage, etc., see Fig 1.1 b. Any change in the target parameters should be visible as a change in vibration frequency or amplitude. That would allow using the analysis of detected frequencies as one of the mechanisms for monitoring the structural health of the target. 


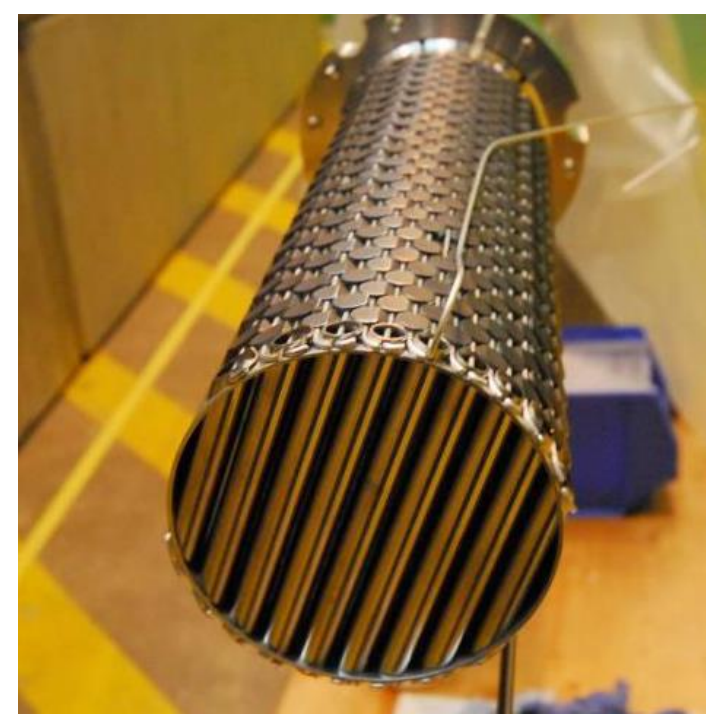

(a)

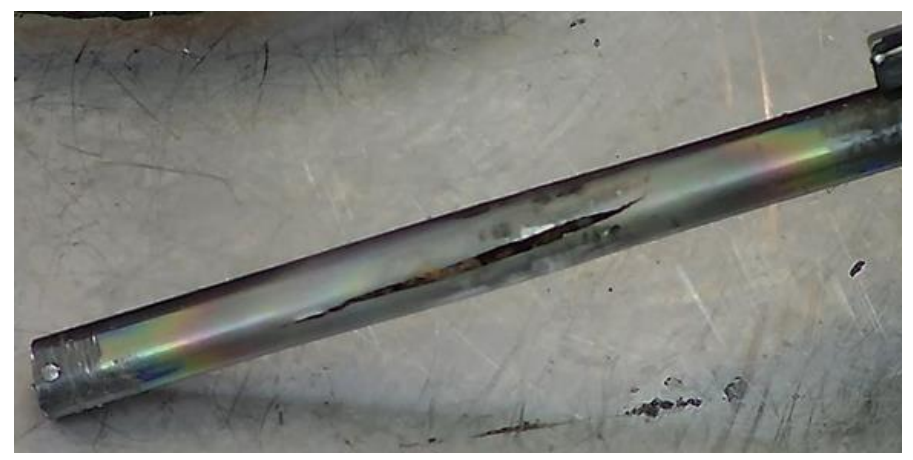

(b)

Fig. 1.1. (a) Mounting of $\mathrm{Pb}$ filled rods in the SINQ proton beam target; (b) Photograph of a rod after an operation in SINQ targets with a damage that leads to mass reduction due to lead leakage [3].

Turbulence-induced vibrations are the result of fluid-structure interaction between a tube and a flow whose turbulence intensity is associated with the level of turbulence of the incoming flow in the tube array, as well as the turbulence caused by upstream rows of rods [4]. Generally, the vibration mechanism caused by turbulence does not cause immediate catastrophic changes to the system but may result in progressive tube damages in the long-term [5].

With the rapid increase in computing capacity and performance, CFD simulations are becoming an increasingly useful approach to flow-induced vibration studies. Although the CFD is a popular and generally accepted practice, obtaining applicable results remains a challenge, for example, there is no single method for modelling or calculating turbulence that is applicable to all cases.

The relatively simplest situation is when a single cylindrical rod is inserted into the flow. Such simulations may be used to validate the selected methods and models, as both experimental and numerical data are available in the scientific literature on the integral characteristics of a single rod, such as lift and drag coefficients or the Strouhal number.

Cooling plants, heat exchangers or nuclear reactors typically contain arrays of rods or tubes with tens or even hundreds of cylindrical elements. Although computing capacity has increased significantly today, but the costs of simulating turbulent flow tend to be high, therefore attempts are made to reduce the computing area as much as possible. A series of studies have been carried out to determine the minimum number of rows and columns needed to model the array behaviour due to the flow, e.g. [6], [7].

Several studies are available in the scientific literature comparing the results of 2D and 3D models. From the results of Large Eddy Simulations described in [8], it can be concluded that the three-dimensional effects of the flow are relatively minor at low Reynolds numbers $(R e=2840)$. On the other hand, if the Reynolds number is medium or large, as it follows from the Shinde et al. [9] publication, the 3D simulation shows about $30 \%$ increase in the amplitude 
of oscillations in comparison to the two-dimensional case at $R e=60000$. The lift coefficient spectrum in $3 \mathrm{D}$ displays higher frequencies indicating that the turbulence energy increases due to the spanwise direction dynamics. The spectra of the response signal of the cylinder show the same frequency of oscillations in $2 \mathrm{D}$ and $3 \mathrm{D}$ simulations. This confirms theoretical considerations of the fluidelastic instability, as well as experimental observations reporting that the instability mainly appears in the perpendicular direction with respect to the cross flow. Thus, it can be concluded that, under certain conditions, 2D simulations give results of acceptable precision. In addition, it should be noted that the computational domain in 3D cases is substantially larger than in $2 \mathrm{D}$, which significantly increases the required computing resources and time. Since this study mainly analyses frequencies, it appears from the above mentioned that it is more optimal to use a $2 \mathrm{D}$ geometry with an appropriately chosen mass and stiffness coefficient.

In a typical case, in laboratory experiments, the rod vibration in the array is determined by using accelerometers or laser sensors. However, it should be noted that it is not possible to apply these methods to real, working installations, particularly in the case of installations operating under radiation conditions. Mathematical approximations should, therefore, be used in the monitoring of real systems.

In order to predict the typical behaviour of the system by using a low-dimensional representation for a high-dimensional problem, a mathematical approximation may be applied, identified in the literature by the terms "metamodel" or "surrogate model". The use of metamodels makes it possible to effectively understand and assess the interdependence of model input (factors) and output (response) parameters using fast simulation tools. Both physical and numerical experiments can be used to obtain factor-response maps. The design of experiments is an essential step for the efficient development of a metamodel. Central composite designs are often used for fitting a second order response surface [10], while it is most efficient to use space filling plans [11], such as different type of Latin hypercube sampling (LHS) [12], [13] for the planning of computer experiments. An important concept in design of experiments (DoE) is orthogonality. This would lead to the best designs since it would minimize the variance of the regression coefficients [10]. New types of orthogonal DoE are described in source [14].

After selecting the experiment plan and performing the necessary simulations or physical experiments, the second phase of metamodelling is carried out, in which a suitable model of approximation (mathematical) must be selected. The aim of approximation is to obtain an instrument for the analysis of a complex or high-cost system [15]. When compiling the information available in scientific literature, it can be concluded that one of the most popular types of approximation models is the response surface method (RSM) developed by Box \& Wilson [16]. RSM is useful and effective in cases where the number of parameters of the system is low and its behaviour is not highly non-linear [17].

Inverse analysis is a currently relevant topic in modern engineering. Inverse problems are defined as problems where the responses are known, but inputs or the source of the responses have yet to be clarified. In inverse metamodelling, methods of approximation are used to create a pattern of inverse relationship. This creates an inverse relationship between consequences and causes that could be used to monitor systems. 


\section{Laboratory Experiments}

Chapter 2 describes the laboratory experimental equipment, the sensors used, the course of the experiment, the results obtained and their postprocessing. An analysis of the experimental data allowed a series of findings on the behaviour of single flexibly mounted rod inserted into an array of closely deployed rods at different initial conditions.

The experimental facility is located at the PSI and the experiments were conducted in collaboration with PSI colleagues. The apparatus consists of a water contour with a test section containing an array of fixed rods with a single flexible mounted test rod (TR), measuring equipment and sensors. The water contour system is composed of rectangular entrance and exit water channels, water pumps, expansion tanks, flow meter, manometer and the test section of the array. The flow rate in the channel can range from 0 to $2.9-3.0 \mathrm{~L} / \mathrm{s}$. Reynolds number, based on the inflow velocity $U_{\text {in }}$ and cylinder diameter $d$, can reach $16.4 \cdot 10^{3}$ ( $R e=U_{\text {in }} d / v$, where $v$ is the kinematic viscosity of water). During the experiment, the flow rate is gradually changed, reaching the maximum from the minimum in 100 steps.

The test segment with a set of rods consists of a rectangular channel with a cross-section of $30 \mathrm{~mm}^{2} \times 97 \mathrm{~mm}^{2}$, which rapidly passes into a smaller cross-section of $30 \mathrm{~mm}^{2} \times 47 \mathrm{~mm}^{2}$. An array of rods with 21 rows of rigid, cylindrical stainless-steel cylinders, is placed in the narrower part of the channel, $97 \mathrm{~mm}$ from the entrance of the section. Rods are placed in a triangular arrangement with a low ratio $P / d=1.1$ (see Fig. 2.1). Each row contains five rods or four rods and two half-rods. The rods are rigidly fixed in hollows in the plexiglass covers. All rods are identical, $30 \mathrm{~mm}$ long, with a diameter of $d=8 \mathrm{~mm}$ and with the same mechanical characteristics. The housing natural frequency is expected to be significantly higher than the TR frequency.

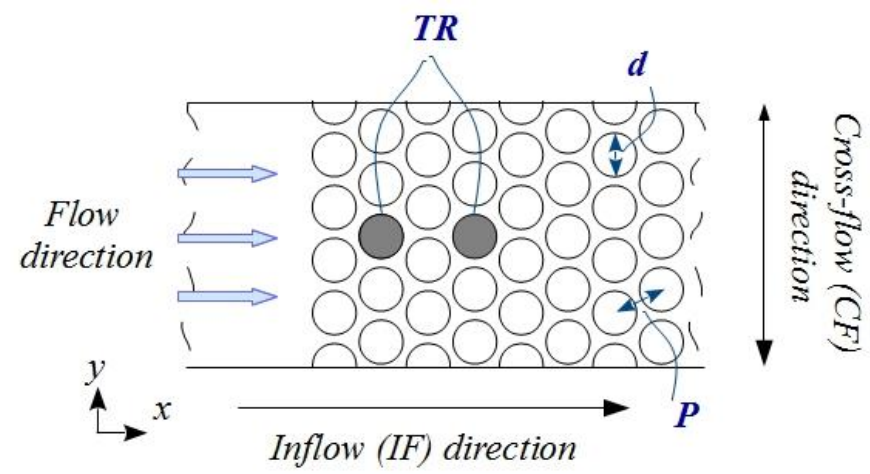

Fig. 2.1. The cross-section for the test section of an array of rods.

Most experimental studies were conducted when TR was in the middle of the fourth row. The TR location was changed from fourth to the second row, with the exception of the first two rows of the array, in order to determine the effect of the TR position in the array and the number of rows of the set. The TR sketch is shown in Fig. 2.2. The ends of TR are embedded in metal bellows, thereby providing waterproofing. The free movement of TR perpendicular 
to the flow (cross-flow, CF) and in the flow direction (inflow, IF) is provided with two elastic cylindrical beams of $128 \mathrm{~mm}$ length. Three elastic beams of different diameters, described by their stiffness coefficient $k$, were used. The mass of the beam is significantly lower than the mass of the rod (less than $3 \%$ of the mass of TR). The effects of TR mass have been studied by experimentally adding mass elements at both ends of the TR. Consequently, the mass of TR was increased by $35 \%$.

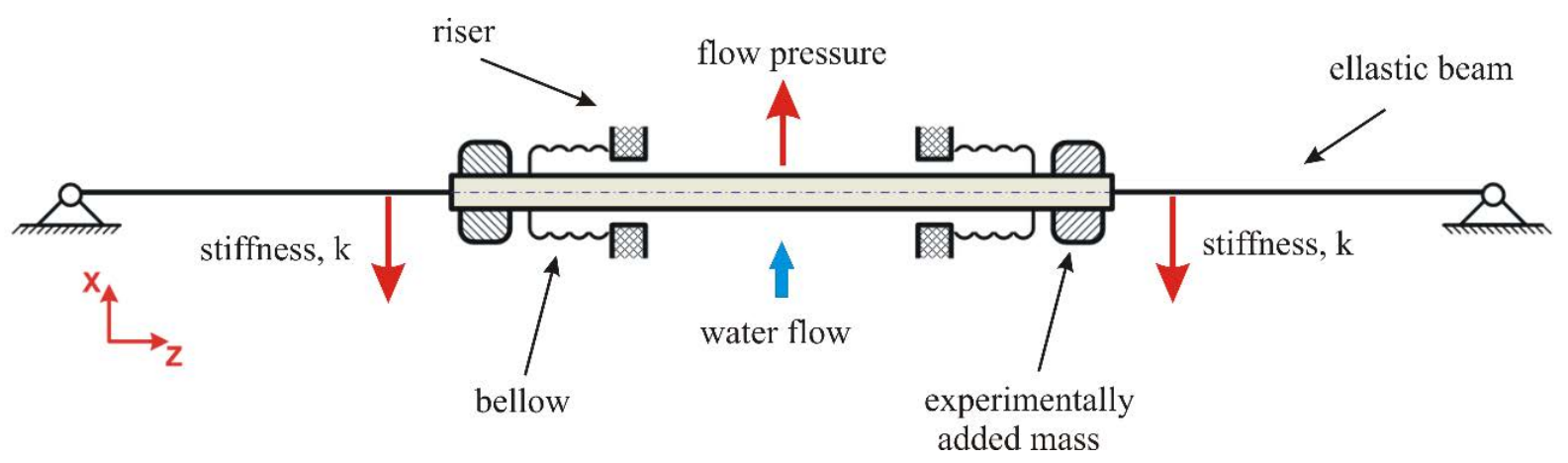

Fig. 2.2. The sketch of flexibly mounted TR.

The set of measurement equipment used includes four single-axis accelerometers and two laser triangulation sensors. The maximum measurement sampling frequency is $2 \mathrm{kHz}$ for both accelerometer and laser sensors. At each end of the TR, there are two accelerometers to obtain TR acceleration measurements in both directions of CF and IF. To obtain the TR velocity and displacement from accelerometer measurement data, the fast Fourier transform (FFT) and the inverse FFT was applied. The laser sensors are located at one end of the TR, one in each direction.

To minimize errors in experimental results, a number of actions were done including repeated measurements with the same initial conditions. Random uncertainties $\left(U_{\mathrm{f}}\right)$ at $95 \%$ confidence limit calculated according the formulation by Reedy [10] are summarized in Table 2.1 where $m^{*}$ is the mass ratio. To check sensors and data acquisition system, the installation with external excitation device, which creates the rod oscillations with known frequency, was build. The impact of different measurement sampling frequencies was checked. The known generated signal was used to check algorithms, which were applied for the postprocessing of the acceleration measurements. The comparison of the displacement from laser sensor measurements and calculated from accelerometer was done as well.

Table 2.1

Random Uncertainties of Frequencies $U_{\mathrm{f}}$ at $95 \%$ Confidence Limit

\begin{tabular}{|l|c|c|}
\hline \multicolumn{1}{|c|}{ Cases } & $f_{\mathrm{n}}, \mathrm{Hz}$ & $U_{\mathrm{f}}, \mathrm{Hz}$ \\
\hline$k_{1}, m^{*}=0.42$ & 41.88 & \pm 0.2040 \\
\hline$k_{2}, m^{*}=0.42$ & 48.09 & \pm 0.1454 \\
\hline$k_{3}, m^{*}=0.42$ & 58.50 & \pm 0.3771 \\
\hline$k_{1}, m^{*}=0.567$ & 38.31 & \pm 0.1301 \\
\hline
\end{tabular}


The experimental studies were started with non-flow measurements. All frequencies detected by sensors in these experiments are considered to be environmental noise. In a typical case, the distribution of the noise measured by accelerometers is similar to Gauss distribution. Experiments were conducted with an array of rods in which all rods are fixed to determine the variables associated with flow or system properties, but not with TR vibrations. In the obtained frequency spectrum, a group with frequencies $f_{\mathrm{t}}$ that depends directly on the flow rate was detected: the faster the water flow, the higher the frequency. The relationship between frequencies and the water flow rate is linear. It may be speculated that the observed frequency group $f_{\mathrm{t}}$ is associated with turbulent oscillations in the water flow and approximately corresponds to the Strouhal number $S t=0.20$.

The total TR displacement was divided into two parts: the permanent deflection $\left(\eta_{p}\right)$ from the starting point of the coordinate system was derived from laser sensor measurements and the average oscillation amplitude $\left(\bar{A}_{\mathrm{f}}\right)$ was obtained from accelerometer data.

The increasing flow can be divided into three areas, depending on the TR vibration amplitude behaviour in relation to water velocity in the corresponding region: 1) amplitudes in the first region are relatively low; 2) in the second region, TR vibrations are increasing rapidly and, when peak value is reached, are decreasing rapidly; 3 ) relatively low amplitudes are observed again in the last region similar to those in the first region.

In cases where the flow rate decreases, peak values for dimensionless vibration amplitudes $\overline{A_{\mathrm{f}}} / d$ are observed at lower flow rates than in the case of increasing flows. The maximum $\bar{A}_{\mathrm{f}} / d$ values in both IF and CF directions are achieved when water flow is increasing. Also, the graph of the permanent deflection when the flow is increasing differs from that of a reducing flow. It follows from the results obtained that a hysteresis effect is observed. Measurements of $\eta_{p}$ show that the zero-point shift in IF direction increases if the flow rate grows. A permanent deviation is also observed in the direction of CF. Since the array configuration is theoretically symmetric, the deviation from the coordinate system's starting point in the direction of CF could be explained by the original TR's unsymmetrical position in the array. The constant deviation of CF increases with an increase in flow rate. Therefore, it can be concluded that the flow rate may increase the inaccuracy of the initial rod position.

The results obtained show that TR mass changes are detectable in the frequency domain. This could be useful for structural health monitoring (SHM) in a situation where the damage to the rod results in a decrease in its mass. Further studies would be needed to identify the minimum variation of TR mass to be detected, which would be visible in the frequency spectrum.

Changes to the rod support were modelled using three types of elastic beams with a stiffness $k_{1}$ (weak), $k_{2}$ (medium), and $k_{3}$ (stiff). The location of the elastic beams can be seen in Fig. 2.2. The maximum values for vibration amplitude in the directions of CF and IF are achieved when beams with medium stiffness $k_{2}$ are used. In order to better understand the behaviour of the TR when the amplitude peak values are reached, the distribution of position $P_{i}(t)$ was introduced, which finds areas in the plane cross-section where the end of TR is most frequently present. 
Two TR movement modes were identified using $\left.P_{i}(t): 1\right)$ TR ends spend most of the time around a point corresponding to a deflection of $\eta_{\mathrm{p}} ; 2$ ) at an interval where the TR natural frequency $f_{\mathrm{n}}$ and $f_{\mathrm{t}}$ have similar values, the end of the rod fluctuates around two equilibrium points (see Fig. 2.3), in addition, the probability graphs are not the same for both ends of the TR. From this, it can be concluded that TR movements have become three-dimensional.

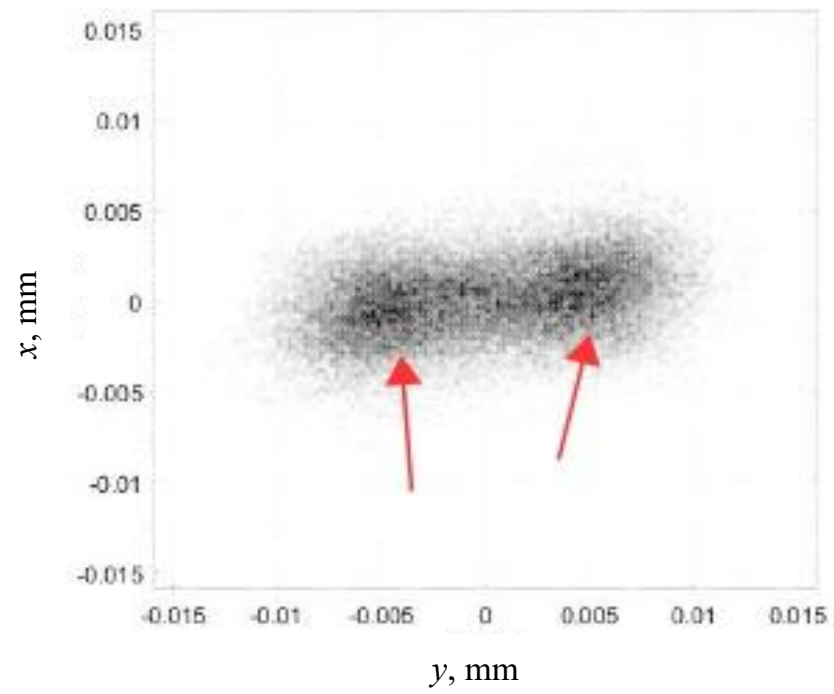

Fig. 2.3. Scatter plot of TR position in time in the $x y$ plane.

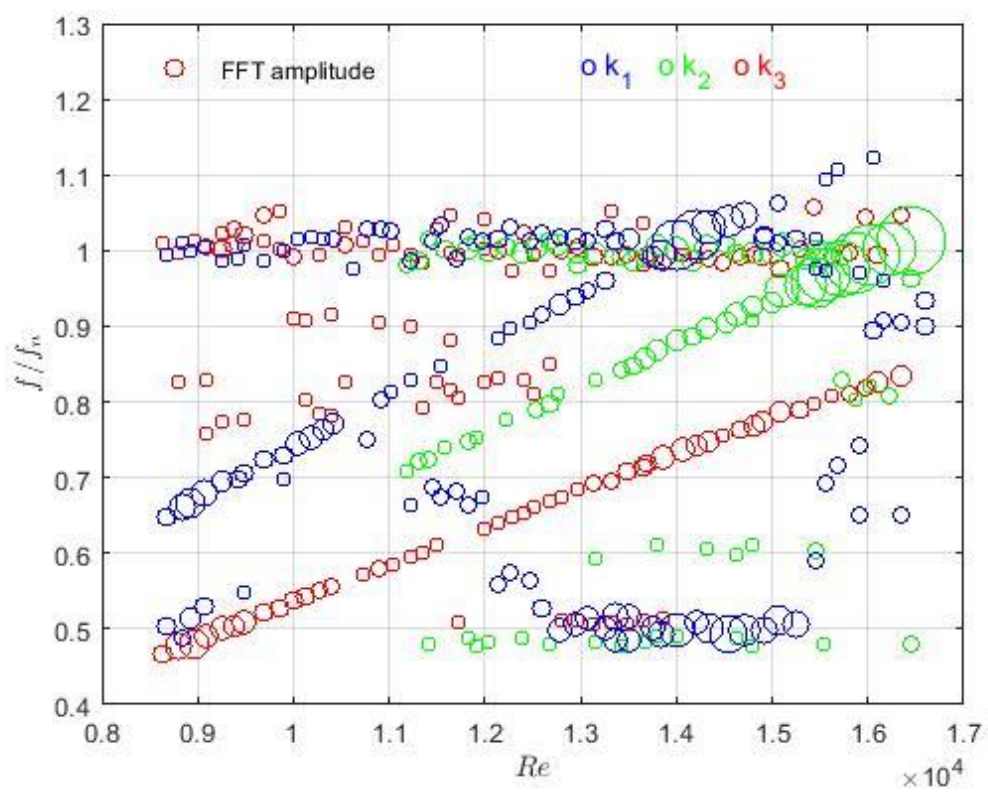

Fig. 2.4. The dominant normalized frequencies in the direction of $\mathrm{CF}$ at the three stiffnesses of elastic beams. The diameter of the circle corresponds to the size of the FFT amplitude. 
In a case where the softest elastic beams with a stiffness $k_{1}$ are used, it can be concluded from the measurement data obtained from the laser sensors that at the reduced velocity $U_{\mathrm{r}}=5.4$ a contact problem begins, i.e. the TR starts to interact with the structure housing. The dominant frequencies for the three stiffnesses of the elastic beam, depending on the Reynolds number, are shown in Fig. 2.4. The diameter of the circles indicates the size of the FFT amplitude. A similar phase portrait is obtained when stiffness is $k_{2}$ and $k_{3}$. When beam stiffness is $k_{1}$, two bifurcation points are observed in the frequency spectrum. If the stiffness of the elastic beam is $k_{1}$ or $k_{2}$, the change in the average oscillation amplitude of the TR may also be detected when changing the position of TR from the fourth row to the second row. In the case with medium stiffness $\left(k_{2}\right)$, the peak value is achieved at the same reduced velocity in both cases, only the maximum value is approximately three times lower if the TR is in the second row.

Analysis of the experimental results obtained shows that the frequency ratio $f_{\mathrm{n}} / f_{\mathrm{t}}$ is more responsive to parameter changes than frequency $f_{\mathrm{t}}$. The results can be seen in Fig 2.5.
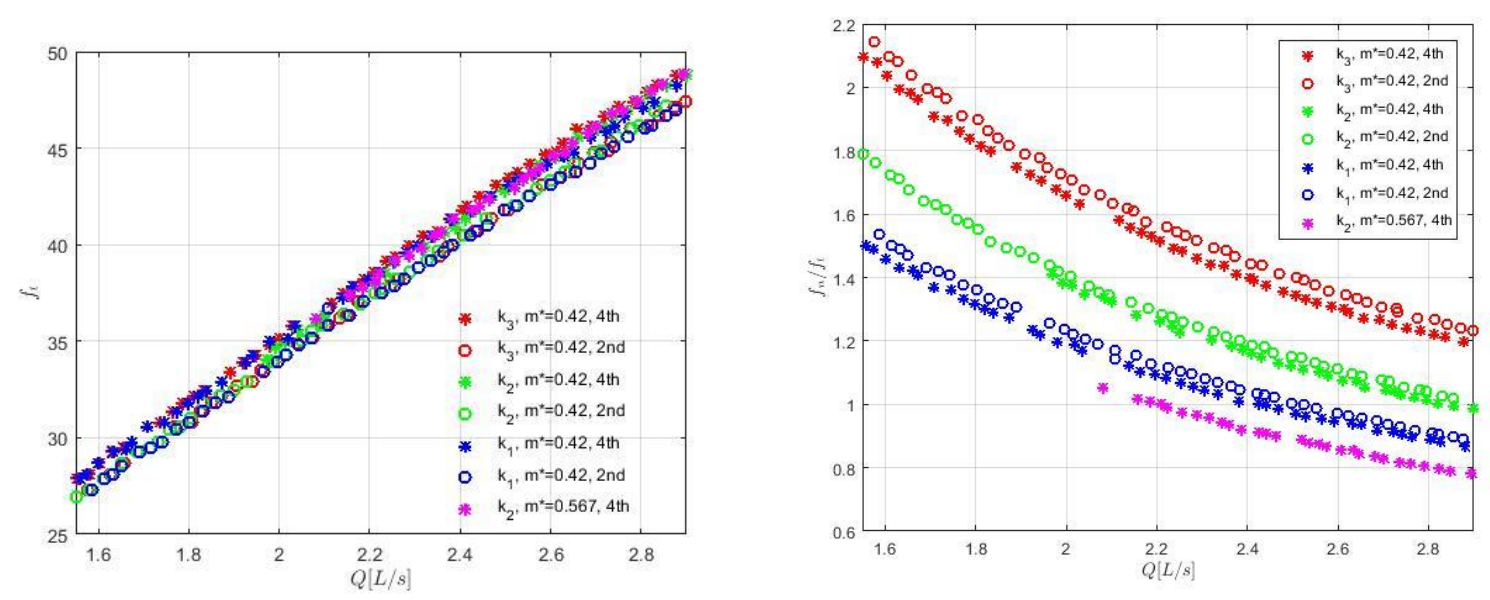

Fig. 2.5. Dominant frequencies depending on the flow rate at different stiffness $k$, rod mass $m$ and TR location in the array. Left - frequency $f_{\mathrm{t}}$. Right - frequency ratio $f_{\mathrm{n}} / f_{\mathrm{t}}$.

\section{Numerical Experiments}

Chapter 3 of the Thesis is devoted to the description of the implemented numerical experiments. Numerical experiments were used to study the flow-induced forces that act on the TR in the rod array and to analyse the mass and stiffness coefficient impact on the TR oscillation frequencies. An additional set of simulations was devoted to reducing computational time and costs as much as possible.

The open-source CFD tool OpenFOAM 2.4.x (OF) was used to solve differential equations of mass and momentum. RANS (Reynolds-Averaged Navier-Stocks) and URANS (Unsteady RANS) equations were solved using the standard k-epsilon and k-omega SST turbulence models to ensure the closure of the equation system. Both low- and high-Reynolds turbulence models were used. Depending on the turbulence model different computational 
domains were analysed. Examples can be seen in Fig. 3.1 where (A) corresponds to the highReynolds turbulence model and (B)-(E) were used with the low-Reynolds model.

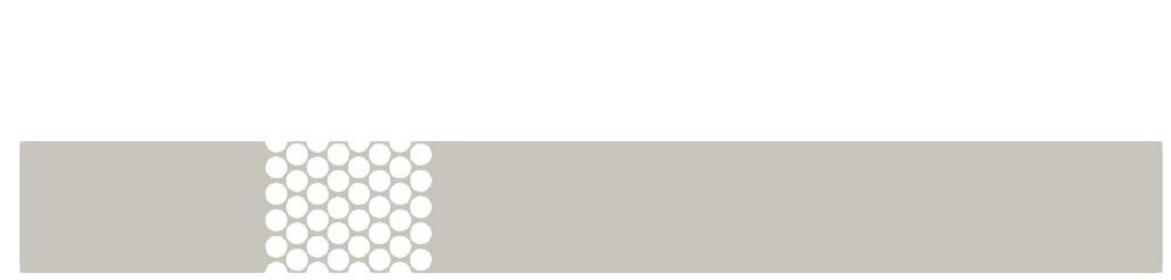

(A)

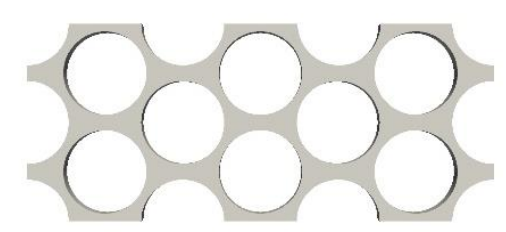

(C)

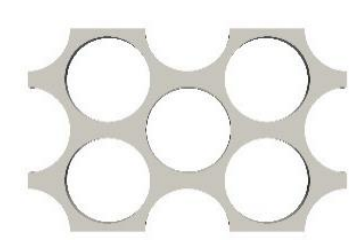

(D)

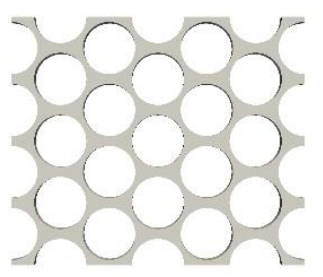

(B)

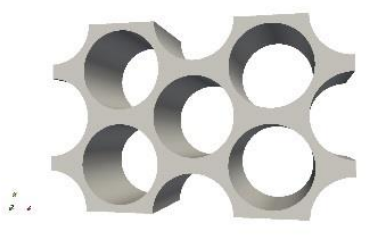

(E)

Fig. 3.1. Examples of analysed computational domain configurations. The flow direction is from left to right. (A) is for a high-Reynolds turbulence model and contains 2.67 million cells.

(B) contains 2.2 million cells, (C) contains 730000 and (D) with 476000 cells are for lowReynolds models. (E) is a 3D low-Reynolds model with 1.7 million cells.

According to Roache [18], the quantification of uncertainty in numerical simulations contains the three most important items: verification of codes, verification of calculations and validation. Since standard OF solvers were used in the modelling process, the verification of the code was not carried out in this case. Solution verification was realized using flow simulation around a single cylinder at $R e=10630$ and comparing integral parameters (Strouhal number, drag and lift coefficients) with data available in the literature. The analysis of the test case allows selecting optimal turbulence model, boundary conditions and computational cell size. Particular attention was paid to the correct description of the boundary layer around cylinders, the final version of which contains 25 layers with an expansion ratio of 1.05 with $y^{+} \leq 1$ and $y^{+} \approx 50$ for low- and high-Reynolds turbulence models, respectively.

Low-Reynolds turbulence model allows predicting the small structures around cylinders, which leads to an increase in the accuracy of hydrodynamic force prediction. The example of the flow separation from the rod is shown in Fig. 3.2. 


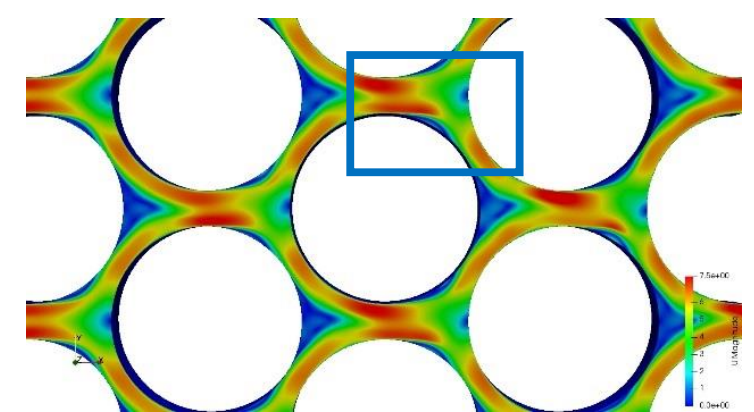

$\mathrm{t}_{1}$

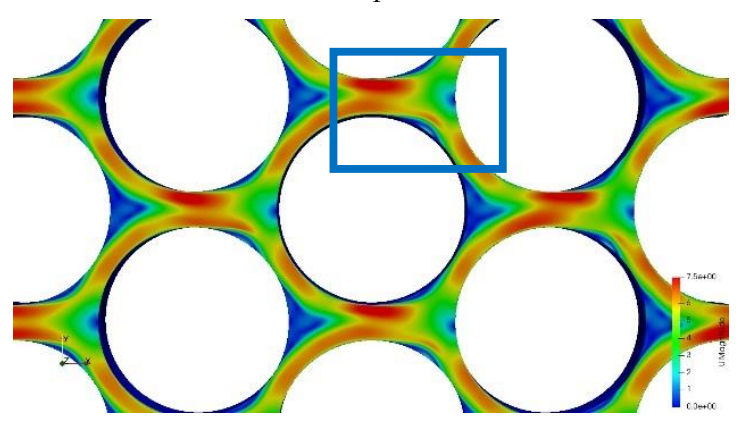

t3

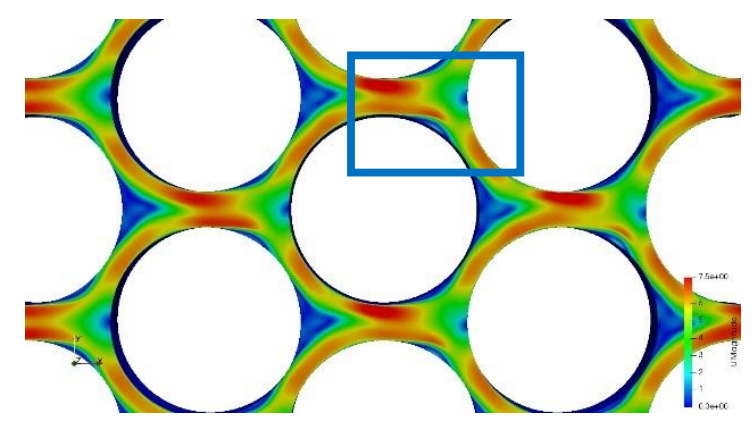

$\mathrm{t}_{2}$

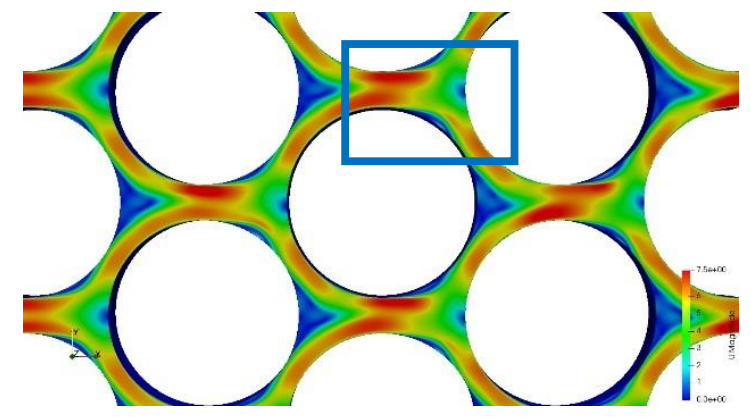

$\mathrm{t}_{4}$

Fig. 3.2. Example of the flow separation from the cylinder during the time.

When comparing the flow rate breakdown between two adjacent cylinders at different input velocities, it can be seen that the velocity profile is in all cases similar, see Fig. 3.3, which indicates that it can be described with an analytical function. Thus, in order to minimise the time required for the calculation, the resulting profile may be assigned as an input parameter for CFD simulations, and the reduced domains (B)-(E) described above may be used. An approximated flow velocity profile in the gap can be obtained by the Fourier series expansion up to the sixth term (3.1). It should also be noted that the flow profile in the gap is time dependent.

$$
\begin{gathered}
\hat{u}(y, t)=C_{1} \frac{4}{\pi} \sin (\omega y+\varphi(t))+C_{2} \frac{4}{3 \pi} \sin (3 \omega y)+C_{3} \frac{4}{5 \pi} \sin (5 \omega y) \\
C_{4} \frac{4}{7 \pi} \sin (7 \omega y)+C_{5} \frac{4}{9 \pi} \sin (9 \omega y)+C_{6} \frac{4}{11 \pi} \sin (11 \omega y),
\end{gathered}
$$

where $\omega$ is angular frequency, $C_{i}=c_{i} U_{\text {in }}$ and $\varphi(t)=A \sin \left(2 \pi f_{\mathrm{t}} t\right)$ is time-dependent component where $A$ is the amplitude and $t$ is time. $A$ and $f_{\mathrm{t}}$ are known from laboratory experiments. $c_{i}$ coefficients were found when analysing the resulting profiles, see Fig. 3.3. Multiplying $c_{i}$ with the inlet velocity $U_{\text {in }}$, the corresponding constants $C_{i}$ can be obtained for any inlet velocity when Reynolds number is between $11.0 \cdot 10^{3}$ and $53.0 \cdot 10^{3}$. The coefficients found are summarized in Table 3.1.

Table 3.1

Coefficients for Obtaining the Velocity Profile Using the Fourier Series Expansion

\begin{tabular}{|c|c|c|c|c|}
\hline Coefficients & $c_{1}$ & $c_{2}$ & $c_{3}, c_{4}$ & $c_{5}, c_{6}$ \\
\hline Value & 1.07 & 1.21 & 0.58 & 0.2 \\
\hline
\end{tabular}




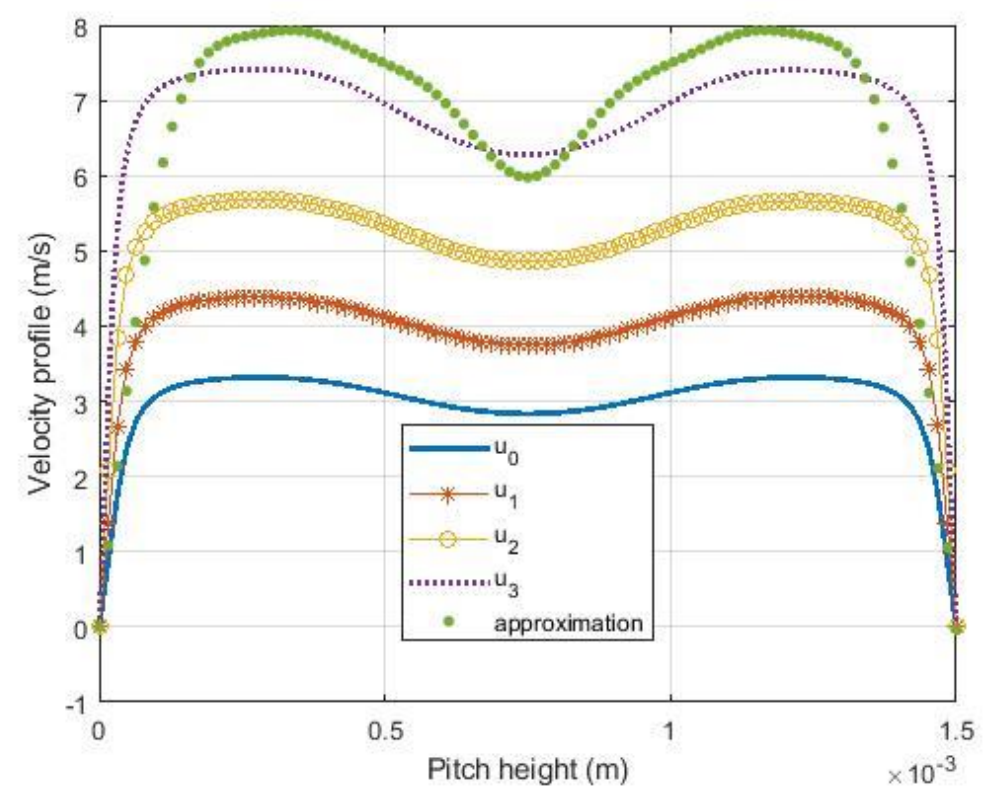

Fig. 3.3. Examples of flow velocity profiles in the gap at four input velocities $\left(u_{0}-u_{3}\right)$ and approximated flow profile for velocity $u_{3}$.

An example of the velocity determined by the Fourier series expansion for velocity $u_{3}$ can be seen in Fig. 3.3 as green dots. Although the graphs vary slightly, the difference in integral value is within $1 \%$ in all cases considered.

Validation of the numerical model with introduced boundary conditions (3.1) for the inlet velocity was realised comparing numerical results with experimental values of force coefficient in the flow direction, $c_{\mathrm{d}}=2 F_{x} /\left(\rho U^{2} S\right)$ and dominant frequencies. The obtained results are summarized in Table 3.2, including force coefficients if the TR is located in the $2^{\text {nd }}$ (domain (D)) and $4^{\text {th }}$ (domain (C)) row. As can be seen in Table 3.2, the obtained CFD results fit well with experimental measurements; the difference is less than $5 \%$. Validation of the high-Reynolds turbulence model was performed using comparison of pressure drop in the water channel.

Table 3.2

Validation of the Numerical Models (Low-Re Turbulence Models)

\begin{tabular}{|c|c|c|c|c|}
\hline & $f_{\mathrm{n}}$ & $f_{\mathrm{t}}$ & $c_{\mathrm{d}}, 2^{\text {nd }}$ row & $c_{\mathrm{d}}, 4^{\text {th }}$ row \\
\hline Experiment & 58.50 & 27.30 & 0.346 & 0.277 \\
\hline CFD, 2D & 59.32 & 28.66 & 0.352 & 0.276 \\
\hline CFD, 3D & - & 28.56 & 0.359 & - \\
\hline
\end{tabular}

The effect of the TR position on the flow-induced forces in the unit cell was analysed by moving the TR by $5 \%$ of the $d$ in the direction of flow and by turning it around the origin of the coordinate system. Given the symmetry of the rod array, the rotation angle is between $0^{\circ}$ and $180^{\circ}$ with an increasing step of $45^{\circ}$. The obtained force coefficients $c_{\mathrm{d}}$ and $c_{1}=$ $2 F_{y} /\left(\rho U^{2} A\right)$ at different Reynolds numbers are summarized in Table 3.3. A schematic 
representation of TR modified positions compared to the original is shown in sketches. TR's original position is in all cases represented as a blue dotted circle. Position P0 corresponds to the case where TR is in the centre of the unit cell. In the case of P1 (circle with continuous line), TR is moved in the direction of flow by $1.25-5 \%$ of $d$. P2 case can be obtained by turning P1 by $45^{\circ}$ around the centre of the unit cell. In a similar way, other cases can be obtained.

Table 3.3

Lift and Drag Coefficients Depending on the TR Position in the Bundle and Flow Velocity

\begin{tabular}{|c|c|c|c|c|}
\hline Position & $R e$ & $c_{\mathrm{d}}$ & $c_{1}$ & Sketch of TR position \\
\hline $\mathrm{P} 0$ & 5600 & 0.006 & 0.0 & \multirow{5}{*}{$\stackrel{\substack{\text { Flow } \\
\text { direction }}}{\longrightarrow}$} \\
\hline $\mathrm{P} 0$ & 11000 & 0.019 & 0.0 & \\
\hline P0 & 17000 & 0.039 & 0.0 & \\
\hline $\mathrm{P} 0$ & 53000 & 0.268 & 0.0 & \\
\hline $\mathrm{P} 0,3 \mathrm{D}$ & 53000 & 0.293 & 0.0 & \\
\hline $\mathrm{P} 1, r=0.05 d$ & 5600 & 0.013 & 0.000 & \multirow{5}{*}{$\begin{array}{c}\text { Flow } \\
\text { direction }\end{array}$} \\
\hline $\mathrm{P} 1, r=0.05 d$ & 11000 & 0.046 & 0.000 & \\
\hline $\mathrm{P} 1, r=0.05 d$ & 17000 & 0.099 & 0.000 & \\
\hline $\mathrm{P} 1, r=0.0125 d$ & 53000 & 0.352 & 0.012 & \\
\hline $\mathrm{P} 1, r=0.0184 d$ & 53000 & 0.408 & 0.008 & \\
\hline $\mathrm{P} 2, r=0.05 d, \alpha=45^{\circ}$ & 5600 & 0.010 & -0.0008 & \multirow{4}{*}{$\begin{array}{c}\text { Flow } \\
\text { direction }\end{array}$} \\
\hline $\mathrm{P} 2, r=0.05 d, \alpha=45^{\circ}$ & 11000 & 0.035 & -0.0017 & \\
\hline $\mathrm{P} 2, r=0.05 d, \alpha=45^{\circ}$ & 17000 & 0.075 & -0.0027 & \\
\hline $\mathrm{P} 2, r=0.05 d, \alpha=45^{\circ}$ & 53000 & 0.601 & 0.0059 & \\
\hline $\mathrm{P} 3, r=0.05 d, \alpha=90^{\circ}$ & 5600 & 0.006 & -0.0007 & \multirow{4}{*}{$\begin{array}{c}\text { Flow } \\
\text { direction }\end{array}$} \\
\hline $\mathrm{P} 3, r=0.05 d, \alpha=90^{\circ}$ & 11000 & 0.021 & -0.0013 & \\
\hline $\mathrm{P} 3, r=0.05 d, \alpha=90^{\circ}$ & 17000 & 0.043 & -0.0016 & \\
\hline $\mathrm{P} 3, r=0.05 d, \alpha=90^{\circ}$ & 53000 & 0.305 & 0.0396 & \\
\hline $\mathrm{P} 4, r=0.05 d, \alpha=135^{\circ}$ & 5600 & 0.005 & 0.0013 & \multirow{4}{*}{$\begin{array}{c}\text { Flow } \\
\text { direction }\end{array}$} \\
\hline $\mathrm{P} 4, r=0.05 d, \alpha=135^{\circ}$ & 11000 & 0.014 & -0.005 & \\
\hline $\mathrm{P} 4, r=0.05 d, \alpha=135^{\circ}$ & 17000 & 0.028 & -0.011 & \\
\hline $\mathrm{P} 4, r=0.05 d, \alpha=135^{\circ}$ & 53360 & 0.137 & -0.0997 & \\
\hline $\mathrm{P} 5, r=0.05 d, \alpha=180^{\circ}$ & 5600 & 0.005 & 0.0 & \multirow{3}{*}{$\begin{array}{c}\text { Flow } \\
\text { direction }\end{array}$} \\
\hline $\mathrm{P} 5, r=0.05 d, \alpha=180^{\circ}$ & 11000 & 0.014 & 0.0 & \\
\hline $\mathrm{P} 5, r=0.05 d, \alpha=180^{\circ}$ & 17000 & 0.026 & 0.0 & \\
\hline
\end{tabular}


Due to the symmetry of the array of rods, the flow-induced force in the transverse $F_{y}$ at the P0 position is zero. The total normalized force $\hat{F}$ contains only force components in the flow direction $F_{x}$. Similarly, in P1 and P5 positions, the total flow-induced force is equal to $F_{x}$. A comparison of the positions of $\mathrm{P} 1$ and $\mathrm{P} 5$ allows assessing the effect of the distance of TR from the previous cylinder on the force. Reducing the distance from the previous cylinder decreases the force as well. In contrast, the increase in the distance leads to an increase in the force. The numerical investigation has shown that the TR position in the flow direction is critical to accurately predict hydrodynamic forces. The $F_{y}$ component will appear when the unit cell becomes unsymmetrical, see positions P2, P3 and P4. A small downward force component can be observed in $\mathrm{P} 2$ when the Reynolds number is small or medium. When the flow rate in the gap is high, the $F y$ component becomes positive, which corresponds to the experimental observations. A similar situation may be observed in the P3 position. A more pronounced force component in the transverse direction $\left(F_{y}\right)$ is observed in the case of $\mathrm{P} 4$. Table 3.3 allows to explain and confirm the experimental observation that at high flow velocity the rod moves mainly in a direction perpendicular to the flow.

The mathematically analysed TR movement resulting from the cross-flow may be described as the mass-spring-damper system:

$$
M \ddot{r}+C \dot{r}+K r=R,
$$

where $\boldsymbol{r}$ is the vector of generalized coordinates $\left(\boldsymbol{r}^{\mathrm{T}}=[x ; y]\right) ; \boldsymbol{R}$ is generalized forces $\left(\boldsymbol{R}^{\mathrm{T}}=\right.$ $\left.\left[F_{x} ; F_{y}\right]\right)$; the dot denotes time derivative.

Matrices $\boldsymbol{M}, \boldsymbol{C}$ and $\boldsymbol{K}$ contain $T R$ mass, damping and stiffness coefficients, respectively. Since the excitation force $\boldsymbol{R}$ is not known, the CFD approach is used to quantify the corresponding force values, considering both the fluid and structural impact.

The flow-induced oscillation of the TR was modelled using built-in six degree-of-freedom solver. Motion in $x y$ plane without rotation was allowed for cases with high-Reynolds turbulence model. To reduce the computing time in the case of low-Reynolds turbulence model, the TR initial position in the direction of flow was changed, according to the results of the experiments, and then simulated oscillations of the rod in a direction perpendicular to the flow.

\section{Approximations}

Chapter 4 describes the methodology for the development of approximation models for assessment of the effects of the system parameters, as well as inverse models, developed using the results of laboratory and numerical experiments. Two factors have been selected for approximation to describe the rod damage: rod mass and rod support stiffness.

RSM (response surface method) was used for approximation. Given that due to the complexity of numerical modelling, noise in CFD results is unavoidable [19], a second-order polynomial was used as the response surface, based on the conclusions of [20] regarding its resilience to the dispersal of results, i.e., noise. 
EDAOpt software [15], [21], developed in the RTU Machine and Mechanism Dynamics Research Laboratory (MMDRL), was used for the development of the design of experiments and approximation of models. In this Thesis, LH experiment plans from EDAOpt with two or three factors were used. The response surface methodology developed in MMDRL differs from others, with LH-type plans being optimized using mean square error (MSE). The 13point orthogonal plan developed by Professor J. Auziňš, which is a part of a new class of design of experiments for use with orthonormal Legendre polynomial [14], was used as well.

The following quality measures have been used to determine the approximated model adequacy: the relative leave-one-out cross-validation error (4.1) [22] and the adjusted coefficient of determination $R^{2}(4.2)$.

$$
\begin{gathered}
\sigma_{\mathrm{cr} \%}=\frac{\sqrt{\frac{1}{N} \sum\left(\hat{y}_{-i}-y_{i}\right)^{2}}}{\sqrt{\frac{1}{N-1} \sum\left(y_{i}-\bar{y}\right)^{2}}} \cdot 100 \% ; \\
R^{2}=1-\frac{N-1}{N-p-1} \frac{\sum\left(\hat{y}_{-i}-y_{i}\right)^{2}}{\sum\left(y_{i}-\bar{y}\right)^{2}},
\end{gathered}
$$

where

$\hat{y}_{-i}-$ expected approximation without taking into account the sampling point $i$ ( $i=$ $1,2, \ldots, N)$

$\bar{y}$ - the mean value;

$N$ - the number of points;

$p$ - the number of fitting coefficients.

To evaluate the goodness of fit, Pearson's chi-squared test is used. Analysis of Variance (ANOVA) shall be used to determine whether sampling dispersions are statistically significant.

An approximation model capable of detecting changes in rod support stiffness in indirect way was developed using laboratory experimental data. The direct model contains two factors: flow rate $Q$ and elastic beam stiffness coefficient $k$. Frequency ratio $f_{\mathrm{n}} / f_{\mathrm{t}}$ was selected as the response, based on the results of laboratory experiments, which show that the frequency ratio is the parameter that best reflects changes in the mass, stiffness coefficient and geometry. The sampling points selection used a central composite design (CCD) with a triple midpoint was created. CCD is one of the classic approaches to physical experiments [23] for the second-order approximations. A second-order regression model was used for approximation, which gives of $8.41 \%$ relative cross-validation error and $R^{2}=0.998$. The Pareto chart shows that the frequency ratio is influenced by both factors: the flow rate and the stiffness coefficient.

The inverse approximation model had two inputs: flow rate $Q$ and frequency ratio $f_{\mathrm{n}} / f_{\mathrm{t}}$. The stiffness coefficient $k$ was used as a response. The experimental points for the inverse model are shown in Fig. 4.1. The second-order response surface model was used as an approximation with a cross-validation error of $3.78 \%$ and $R^{2}=0.999$. The adequacy of the model is confirmed by Pearson's chi-squared test as well. 
The inverse model for the stiffness of the rod support as a function of the flow rate and frequency ratio may be composed as:

$$
\begin{gathered}
k\left(Q, \frac{f_{\mathrm{t}}}{f_{\mathrm{n}}}\right)=-88736 Q-201528 \frac{f_{\mathrm{t}}}{f_{\mathrm{n}}}+8457 Q^{2}+52780 Q \frac{f_{\mathrm{t}}}{f_{\mathrm{n}}} \\
+44664\left(\frac{f_{\mathrm{t}}}{f_{\mathrm{n}}}\right)^{2}+203522 .
\end{gathered}
$$

Figure 4.2 shows the approximation model of the stiffness obtained by EDAOpt software.

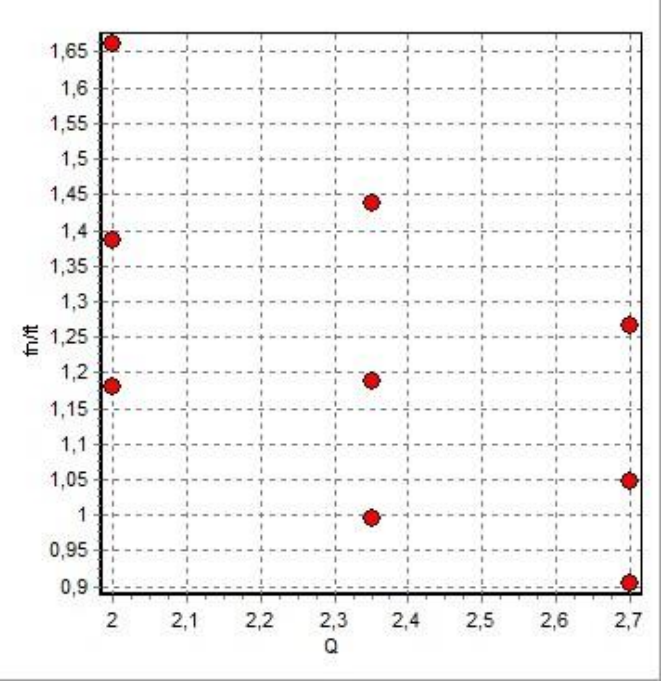

Fig. 4.1. Experimental points for the inverse model of stiffness.

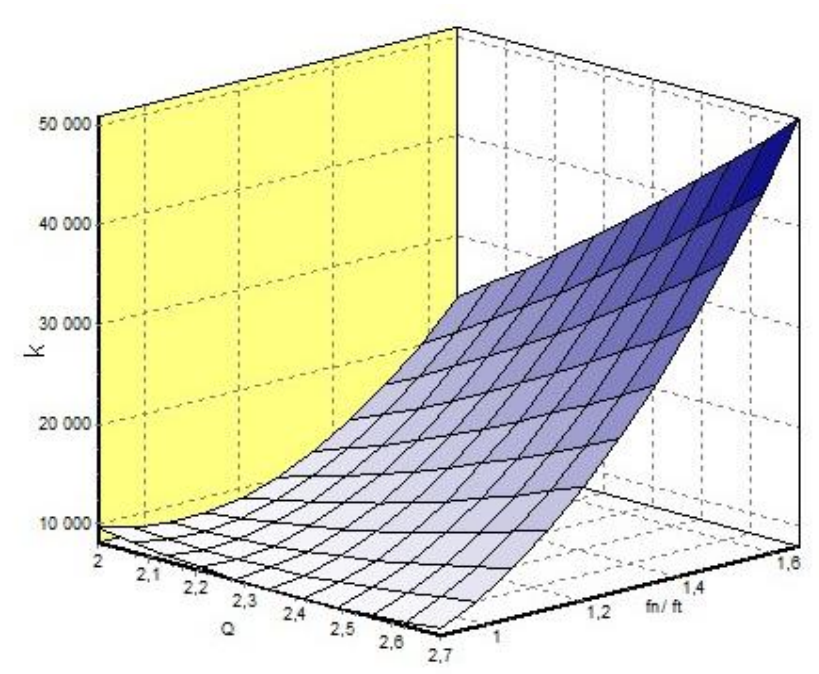

Fig 4.2. The approximation model of stiffness $k$ versus design parameters $Q$ and $f_{\mathrm{n}} / f_{\text {t. }}$.

The validation of the inverse model was realised choosing two constant flow rates and comparing model results with values of the laboratory experiments at these flow rates. Graphs of the stiffness coefficient as a function of frequency ratio using approximation model (4.3) and data from experiments when the flow rate is $2.1 \mathrm{~L} / \mathrm{s}$ and $2.5 \mathrm{~L} / \mathrm{s}$ are shown in Fig. 4.3. One can see that the resulting model is well in line with the laboratory experiments. The maximum difference between experimental and model values is less than $3.4 \%$. 

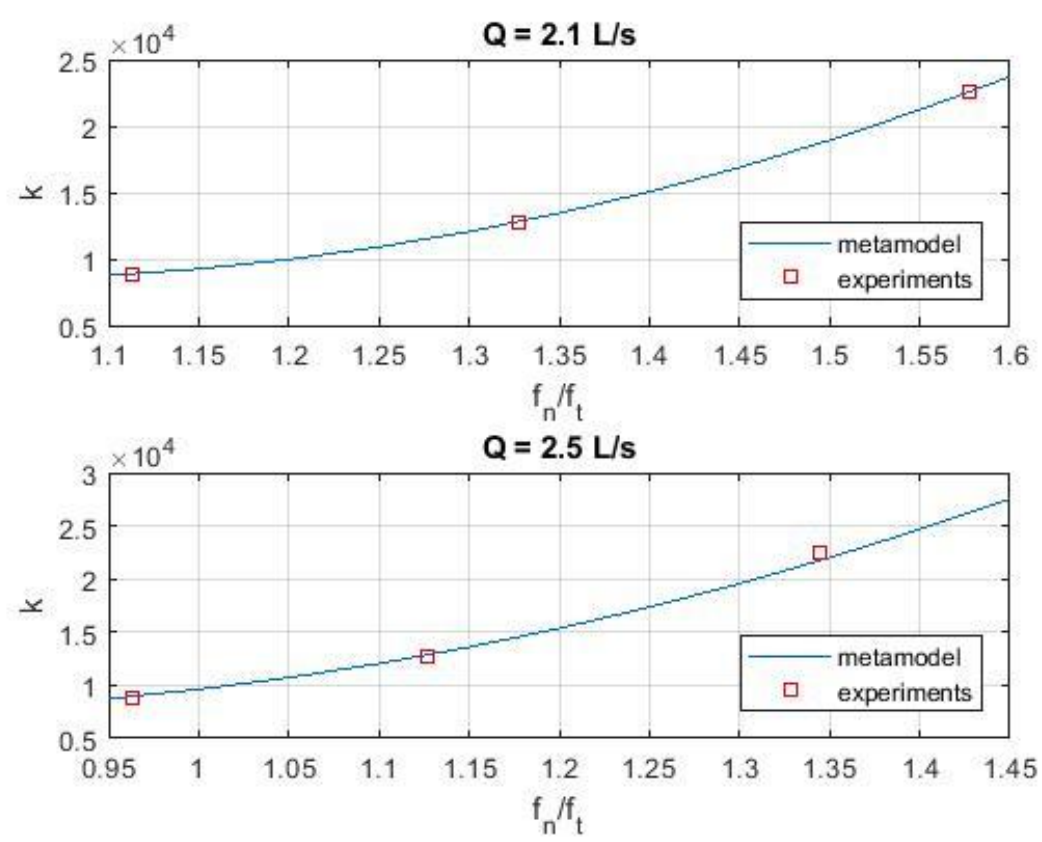

Fig. 4.3. Validation of the inverse model if $Q$ is $2.1 \mathrm{~L} / \mathrm{s}$ and $2.5 \mathrm{~L} / \mathrm{s}$.

CFD simulations were used to acquire a metamodel that would indirectly detect a change in the mass of the rod. A 13-points orthogonal plan was used to create the metamodel of the mass and stiffness as factors and frequency ratio $f_{\mathrm{n}} / f_{\mathrm{t}}$ as a response. Unlike classical CCD plans, in this case, all plan points are within the research area, see Fig. 4.4.

Relative cross-validation error versus number of terms

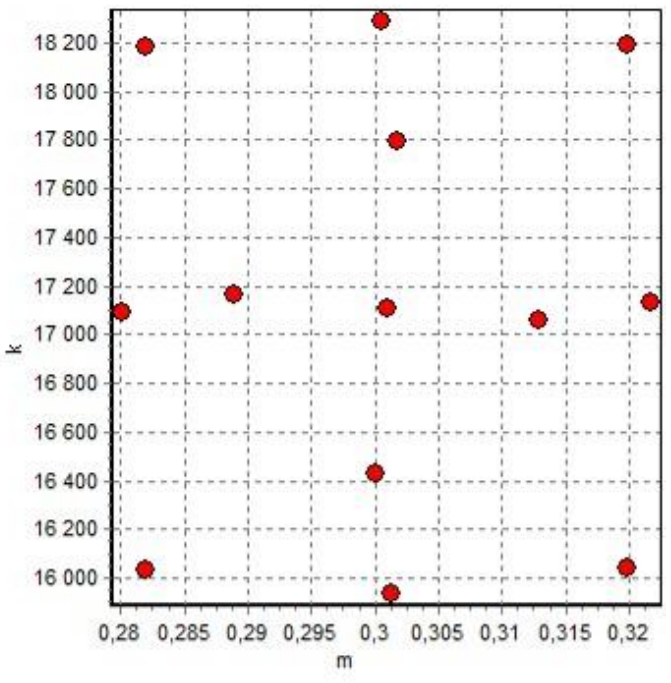

Fig. 4.4. A 13-points orthogonal plan.

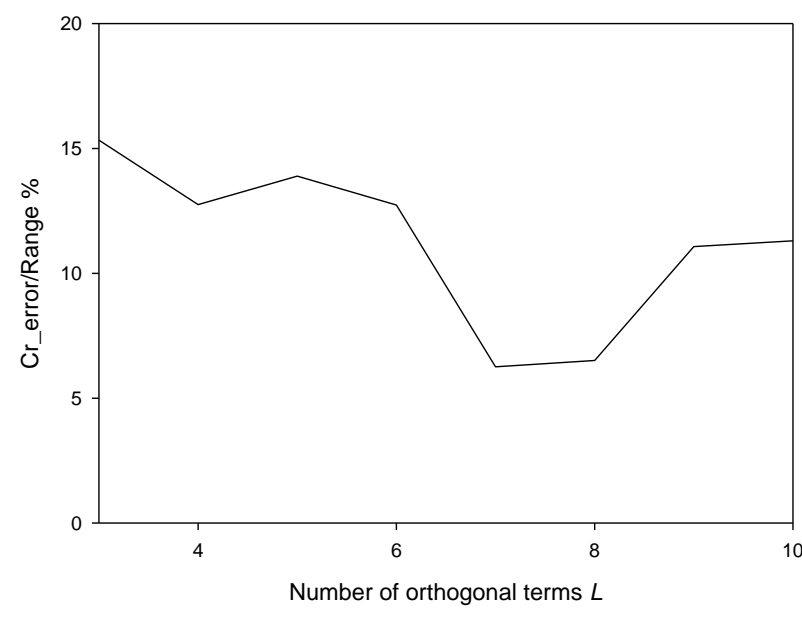

Fig 4.5. The relative cross-validation error depending on the number of terms of approximation using Legendre polynomials.

The initial position of the TR in the direction of flow was first adjusted according to the results of the laboratory experiments. Then simulations were performed in which the TR could only move perpendicular to the flow. The best approximation was obtained using a third-order polynomial with the following quality indicators: $R^{2}=0.994$ and the relative 
cross-validation error $29.68 \%$. Because the experiment plan is orthogonal, Legendre polynomials can be used as an approximation. The property of orthogonality gives the possibility of optimal selection of significant terms in the approximation polynomial function [14]. The relative cross-validation error depending on the number of terms of the polynomials is presented in Fig. 4.5. From Fig. 4.5, it follows that the lowest error is obtained by a 7-term Legendre polynomial.

The inverse metamodel for predicting the mass changes was created using $f_{\mathrm{t}} / f_{\mathrm{n}}$ and $k$ as factors. Of the options examined, the best approximation was achieved by the use of a second-order response surface with the following quality measures: $9.288 \%$ cross-validation error and $R^{2}=0.999$. Pearson's chi-squared test also showed that the model is adequate. The obtained model equation is

$$
\begin{aligned}
m\left(\frac{f_{\mathrm{t}}}{f_{\mathrm{n}}}, k\right)= & -1.37116 \frac{f_{\mathrm{t}}}{f_{\mathrm{n}}}-5.35 \cdot 10^{-5} k+0.709378\left(\frac{f_{\mathrm{t}}}{f_{\mathrm{n}}}\right)^{2} \\
& +9.7 \cdot 10^{-5} \frac{f_{\mathrm{t}}}{f_{\mathrm{n}}} k-1.0 \cdot 10^{-10} k^{2} .
\end{aligned}
$$

The obtained inverse model verification was implemented by selecting specific points and comparing results of the model and numerical experiments at these points. The comparison graph is shown in Fig. 4.6. From Fig. 4.6, it can be concluded that the resulting metamodel is able to predict the behaviour of the analysed model system with high precision, when input factor values are changed within $15 \%$ the difference is less than $1 \%$.

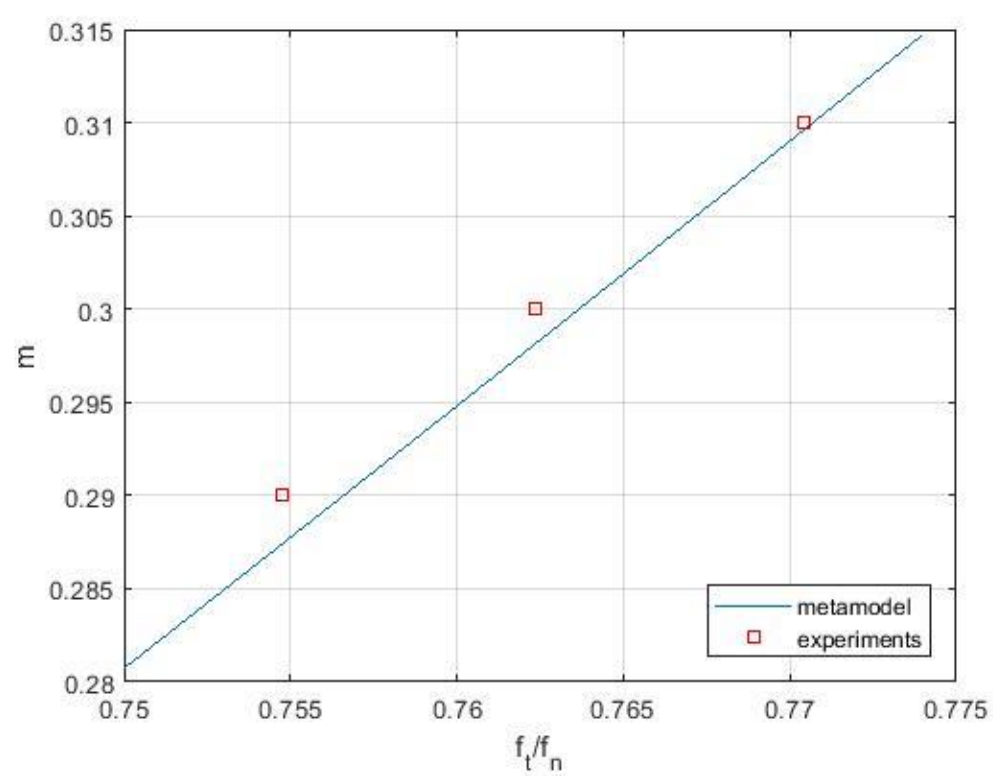

Fig. 4.6. Verification of the inverse metamodel if $k$ is $17600 \mathrm{~N} / \mathrm{m}$.

The analysis carried out shows that the developed method of approximation and the obtained factors-response relationships have a good fit with the laboratory and numerical experiments and therefore it is useful in the monitoring of multi-rods cooling systems as well as in the design process of such systems. 


\section{CONCLUSIONS}

In this Thesis, it was examined how to identify changes to two parameters of the system the mass of the rod and its support stiffness, by analysing the range of vibrations caused by the crossflow of water, if the basic mechanisms that cause vibrations in the system at the given Reynolds numbers are turbulence and vortex shedding. Three methods were used to analyse the dynamics and safety of the water-cooling system: laboratory experiments, numerical hydrodynamics simulations, and approximation. Several conclusions can be drawn when compiling the results of the study.

I. The results of the laboratory experiments obtained show that the rod's natural frequency in the flow and the turbulence induced frequency dependency on the parameters analysed - the geometry of the rods array, the support stiffness of the rod, and the mass and position of the rod in the array, is to be detected in the spectrum. The visibility of changes in system parameters in the frequency domain can be applied to early-stage diagnostics.

II. The analysis of the results shows that in the area where the rod's natural frequency in the flow and the vortex shedding frequency overlaps, increases not only the amplitude of rod oscillations but also the fluctuations around the midpoint of the rod are observed. In the described resonance region, rotational kinetic energy may be up to five times higher.

III. When analysing the results of computational fluid dynamics at different flow rates (before resonance region), it was concluded that in the gap between two adjacent cylinders, the flow rate profile can be described using the Fourier series expansion. These boundary conditions allow an approximate flow profile for any input rate if Reynolds number in the gap is between $11,0 \cdot 10^{3}$ and $53.0 \cdot 10^{3}$. As well the boundary conditions allowed a reduction in the computational domain from 2.2 million to 730000 or 476000 cells, depending on the location of the TR, while maintaining the predicted frequency accuracy of $5 \%$ compared with data from laboratory experiments.

IV. An analysis of the position of the flexibly-mounted cylinder in the unit cell and the dependency of the forces operating on it shows that the deviation of the rod in the direction of flow is essential to obtain the corresponding forces operating on the rod. Consequently, the permanent deflection in the direction of flow from the centre of the cell should be taken into account in the vibration studies where the movement of the rod is allowed only in the direction perpendicular to the flow.

V. When comparing the results of the inverse model, which was designed to obtain the relationship between the frequency ratio (the frequency generated by turbulence and the rod's natural frequency), the flow rate and the stiffness coefficient, with laboratory experiments can be concluded that the stiffness of the rod support can be approximately described by a second-order polynomial with $3.78 \%$ cross-validation error and $R^{2}=0.999$. The resulting model is helpful in cases where major malfunctions are related to changes in rod support stiffness. 
VI. In an analysis of oscillating mass changes detection capabilities using data from CFD simulations, it was found that a qualitative approximation was provided by an inverse metamodel describing the mass as a function of the stiffness of the rod support and frequency ratio. The second-order approximation model with $9.288 \%$ cross-validation error and $R^{2}=0.999$ is applicable to the alleviation of uncertainty, monitoring of cooling systems and early detection of damage associated with a reduction in the mass of the rods.

Directions for further work

I. Assessment of the minimum changes in the mass and stiffness to be detected in frequencies spectrum, so that the methodology is applicable to early diagnosis.

II. Applying of the developed methodology to problems related to the use of the Computational Fluid Dynamics - Discrete Element Method (CFD-DEM). 


\section{REFERENCES}

[1] R. D. Blevins. Flow-Induced Vibration. Krieger Publishing Company, 2001.

[2] M. J. Pettigrew, L. N. Carlucci, C. E. Taylor, N. J. Fisher. Flow-induced vibration and related technologies in nuclear components. Nuclear Engineering and Design, 131(1): 81100, October 1991.

URL http://www.sciencedirect.com/science/article/pii/002954939190319D.

[3] Sobbia R., Dai Y., Jollet S., Wohlmuther M. 3D thermal-structural analyses of SINQ rod bundle target. Proc. of 13th International Topical Meeting on the Nuclear Applications of Accelerators, 306-315, 2017.

[4] R. Alvarez-Briceno, F. T. Kanizawa, G. Ribatski, Leopoldo P.R. de Oliveira. Validation of turbulence induced vibration design guidelines in a normal triangular tube bundle during two-phase crossflow. Journal of Fluids and Structures, 76: 301-318, 2018. URL https://doi.org/10.1016/j.jfluidstructs.2017.10.013.

[5] H. Xu, M. Mallet, T. Liszkai. Turbulent buffeting of helical coil steam generator tubes. In Proceedings of ASME 2014 Pressure Vessels and Piping Conference, page V004T04A075. ASME, 2014.

[6] D.S. Weaver, M. El-Kashlan. On the number of tube rows required to study cross-flow induced vibrations in tube banks. Journal of Sound and Vibration, 75(2): 265-273, 1981. doi: 10.1016/0022-460X(81)90344-8.

[7] D. Tang, S. Bao, M. Xu, L. Luo, B. Lv, L. Yu, H. Cui. On the number of tubes required to study oscillating vortices and frequency spectrums of tube arrays in cross flow. Annals of Nuclear Energy, 124: 198-210, 2019. URL https://doi.org/10.1016/j.anucene.2018.10.008.

[8] Proc. ERCOFTAC Symposium on Unsteady Separation in Fluid-Structure Interaction,2013. ERCOFTA.

[9] V. Shinde, T. Marcel, Y. Hoarau, T. Delozeand G. Harran, F. Baj, J. Cardolacciaand, J.P. Magnaud, E. Longatte, M. Braza. Numerical simulation of the fluid-structure interaction in a tube array under cross flow at moderate and high Reynolds number. Journal of Fluids and Structures, 47: 99-113, May 2014.

URL https://doi.org/10.1016/j.jfluidstructs.2014.02.013.

[10] T. A. Reddy. Applied Data Analysis and Modeling for Energy Engineers and Scientists. Springer, 2011.

[11] T. W. Simpson. A Concept Exploration Method for Product Family Design. PhD thesis, Georgia Institute of Technology, 1998.

[12] P. Audze, V. Eglajs. New approach for planning out of experiments. Problems of Dynamics and Strengths, 35: 104-107, 1977.

[13] J. S. Park. Optimal latin-hypercube designs for computer experiments. Journal of Statistical Planning and Inference, 39(1): 95-111, 1994.

URL https://doi.org/10.1016/0378-3758(94)90115-5.

[14] J. Auziņš. High order orthogonal designs of experiments for metamodeling, identification and optimization of mechanical systems. Proc. 11th World Congress on Computational 
Mechanics, 5th European Conference on Computational Mechanics, and 6th European Conference on Computational Fluid Dynamics, 3190-3201, 2014.

[15] J. Auziņš, A. Januševskis. Eksperimentu Plānošana un Analīze. RTU izdevniecība, 2007.

[16] G. E. P. Box, K.B. Wilson. On the experimental attainment of optimum conditions. Journal of the Royal Statistical Society. Series B, 13(1): 1-45, 1951.

URL https://www.jstor.org/stable/2983966?seq=1\#page_scan_tab_contents.

[17] M. Ramu, R. V. Prabhu. Metamodel based analysis and its applications: a review. Acta Technica Corvininesis - Bulletin of Engineering, 6(3): 25-34, 2013.

URL http://acta.fih.upt.ro/pdf/2013-2/ACTA-2013-2-02.pdf.

[18] P. J. Roache. Verification and Validation in Computational Science and Engineering. Hermosa Publishers, 1998.

[19] J. I. Madsen, W. Shyy, R.T. Haftka. Response surface techniques for diffuser shape optimization. AIAA Journal, 38(9): 1512-1518, 2000. URL https://doi.org/10.2514/2.1160.

[20] R. Jin, W. Chen, T.W. Simpson. Comparative studies of metamodelling techniques under multiple modelling criteria. Structural and Multidisciplinary Optimization, 23(1): 1-13, 2001. URL https://doi.org/10.1007/s00158-001-0160-4.

[21] J. Auzins, A. Janusevskis, J. Janusevskis, E. Skukis. Software EDAOpt for experimental design, analysis and multiobjective robust optimization. Proc. of OPT-i International Conference on Engineering and Applied Sciences Optimization, 101-123, OPT-i, 2014.

[22] J. Auzins, A. Chate, R. Rikards, E. Skukis. Metamodeling and robust minimization approach for the identification of elastic properties of composites by vibration method. Zeitschrift fur Angewandte Mathematik und Mechanik, 95(10): 1012-1026, 2015. URL: https://doi.org/10.1002/zamm.201500008.

[23] D. C. Montgomery. Design and Analysis of Experiments. John Wiley \& Sons, 2017. 